\title{
GEOMETRY AND ALGEBRA OF THE DELTOID MAP
}

\author{
JOSHUA P. BOWMAN
}

\begin{abstract}
The geometry of the deltoid curve gives rise to a self-map of $\mathbb{C}^{2}$ that is expressed in coordinates by $f(x, y)=\left(y^{2}-2 x, x^{2}-2 y\right)$. This is one in a family of maps that generalize Chebyshev polynomials to several variables. We use this example to illustrate two important objects in complex dynamics: the Julia set and the iterated monodromy group.
\end{abstract}

\section{INTRODUCTION.}

Complex dynamics is perhaps best known for the fractal images it produces. For instance, given a polynomial function $\mathbb{C} \rightarrow \mathbb{C}$, an important set to consider is the Julia set, whose points behave "chaotically" under iteration of the function; for most polynomials, the Julia set is a fractal. However, the Julia set is a smooth curve in the case of two special families: power maps, having the form $z \mapsto z^{d}$, and Chebyshev polynomials, of which the simplest example is $z \mapsto z^{2}-2$. For power maps, the Julia set is the unit circle, and for Chebyshev polynomials it is the segment $[-2,2]$, contained in the real line. These structurally simple examples play a distinguished role in complex dynamics, and studying them can illuminate parts of the theory that apply in more complicated cases.

Power maps have an obvious generalization to functions from $\mathbb{C}^{n}$ to itself: just take the $d$ th power of each coordinate. The higher-dimensional analogues of Chebyshev polynomials are not as obvious, however. In the 1980s, Veselov [19, 20] and Hoffman-Withers [7] independently constructed a family of "Chebyshev-like" self-maps of $\mathbb{C}^{n}$ associated to each crystallographic root system of rank $n$. The cases where $n=2$ have received much further attention (see, e.g., [9, 11, 14, 15, 16, 17, 21]), especially for the $A_{2}$ root system, which is connected with the deltoid curve (a.k.a. three-cusped hypocycloid or Steiner's hypocycloid).

This article presents a new approach to construct a quadratic $A_{2}$-type map $f$ based directly on geometric properties of the deltoid. For this reason we call $f$ the deltoid map. The set of lines tangent to the deltoid will play a crucial role, and indeed we will see that $f$ preserves this set of lines. (This fact was previously observed in [21; the difference is that we construct the map from the tangent lines, rather than starting with the map ahead of time and deducing from it the invariance of the tangent lines; in particular, our approach does not use the theory of root systems.) Using this invariance property, we will study two dynamical features of $f$ : one geometric (the Julia set) and the other algebraic (the iterated monodromy group). Both of these objects will be formally defined later in the article.

The Julia set of $f$ is a real algebraic hypersurface $J$ of degree 4 (Corollary 1). We derive this property from a description of $J$ in terms of pedal curves, which arise from classical differential geometry (Theorem 11). The Julia set of $f$ is therefore considerably more interesting geometrically than in the case of a Chebyshev polynomial in one variable, the segment $[-2,2]$ mentioned above.

Date: May 13, 2020. 
The iterated monodromy group of $f$ is an affine Coxeter group (Theorem 2). Such groups are present implicitly in the construction from [19, 20] and explicitly in [7]. The connection with iterated monodromy groups is new, however, and extends the (very short) list of polynomial endomorphisms of $\mathbb{C}^{n}$, with $n \geq 2$, whose iterated monodromy groups are known (see [4, 13. for the only other examples known to the author).

In future work, we will show how these properties of the deltoid map generalize to other Chebyshev-like maps.

\section{LINES AND PLANES.}

In this section we establish some notation and terminology.

The complex projective line $\mathbb{C P}^{1}$ is identified with the one-point compactification of $\mathbb{C}$ (i.e., the Riemann sphere) in the usual way; generally $t \in \mathbb{C} \cup\{\infty\}$ will be used to mean this extended complex coordinate on $\mathbb{C P}^{1}$. The complex projective plane $\mathbb{C P}^{2}$ has homogeneous coordinates $[x: y: z]$, where $x, y$, and $z$ are complex numbers, not all zero; this means that $[x: y: z]=[\alpha x: \alpha y: \alpha z]$ for all $\alpha \in \mathbb{C} \backslash\{0\}$. We use $[a: b: c]^{\vee}$ to represent homogeneous coordinates on the dual projective plane $\left(\mathbb{C P}^{2}\right)^{\vee}$, whose elements are the lines in $\mathbb{C P}^{2}$, so that

$$
[x: y: z] \in[a: b: c]^{\vee} \quad \Longleftrightarrow \quad a x+b y+c z=0 .
$$

The affine plane $\mathbb{C}^{2}$ is canonically included in $\mathbb{C P}^{2}$ via the map $(x, y) \mapsto[x: y: 1]$. The complement of the image of $\mathbb{C}^{2}$ under this embedding is the complex line at infinity $L_{\infty} \cong$ $\mathbb{C P}^{1}$, having equation $z=0$; that is, $L_{\infty}=[0: 0: 1]^{\vee}$.

The real plane in $\mathbb{C}^{2}$ with equation $y=\bar{x}$ is a copy of the Euclidean plane, and it will be denoted by $\mathbb{E}^{2}$. Its closure in $\mathbb{C P}^{2}$ is a copy of the real projective plane, $\overline{\mathbb{E}}^{2} \cong \mathbb{R P}^{2}$, but we do not write it as such, because the coordinates induced on $\mathbb{E}^{2}$ as a subset of $\mathbb{C}^{2}$ are not real. We call $\partial \mathbb{E}^{2}=\overline{\mathbb{E}}^{2} \backslash \mathbb{E}^{2}=\overline{\mathbb{E}}^{2} \cap L_{\infty} \cong S^{1}$ the circle at infinity, trusting no confusion will arise from the fact that the (real) circle at infinity is contained in the (complex) line at infinity.

As a real submanifold of $\mathbb{C P}^{2}, \mathbb{E}^{2}$ does not carry a complex structure (else its closure could not be the real projective plane, topologically), but the restriction of the coordinate $x$ to $\mathbb{E}^{2}$ provides a bijection $\mathbb{E}^{2} \cong \mathbb{C}$. This is what we will always mean when we carry out constructions on $\mathbb{E}^{2}$ using a complex coordinate.

\section{The Deltoid as a ReAl CURVe And AS A COMPlex CuRVE.}

In this section we collect some known properties of the deltoid - especially regarding its tangent lines - that will be useful in our study.
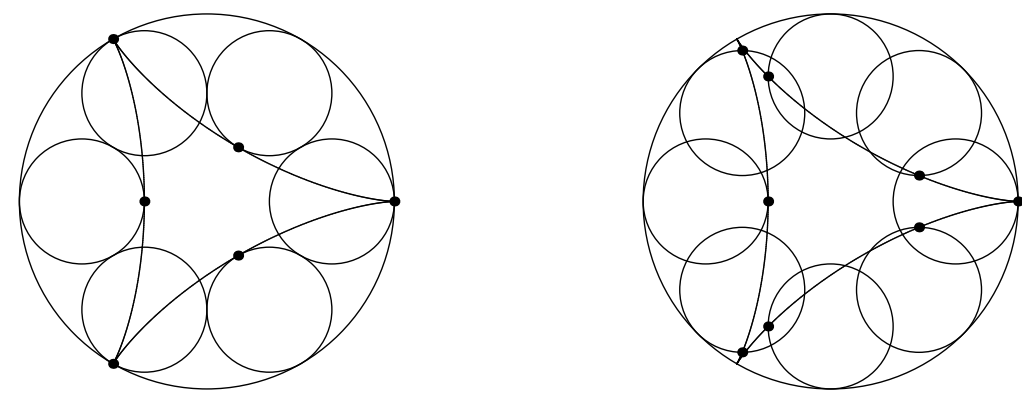

Figure 1. Tracing out the deltoid as a hypocycloid. 
The classical deltoid is the curve traced by a point marked on the circumference of a circle of radius 1 rolling without slipping inside a circle of radius 3 . When the center of the smaller circle travels once counterclockwise around the center of the larger circle, a point on the smaller circle's circumference makes two clockwise revolutions around its center. (See Figure 1.) Because the centers remain 2 units apart, the deltoid can be parametrized in $\mathbb{E}^{2}$ by

$$
x=2 t+\vec{t}^{2}, \quad|t|=1 .
$$

This extends to a complex algebraic curve in the following way. Because $\mathbb{E}^{2}$ is embedded in $\mathbb{C}^{2}$ as the real plane $y=\bar{x}$, the parametrization of the deltoid in $\mathbb{C}^{2}$ becomes $\left(2 t+\bar{t}^{2}, 2 \bar{t}+t^{2}\right)$ with $|t|=1$. In order to make this parametrization holomorphic, we replace $\bar{t}$ with $t^{-1}$ (when $|t|=1$, these are the same), and we define

$$
\gamma(t)=\left(2 t+\frac{1}{t^{2}}, \frac{2}{t}+t^{2}\right), \quad t \in \mathbb{C} \backslash\{0\} .
$$

We can further extend $\gamma$ to a curve in $\mathbb{C P}^{2}$, which we also call $\gamma$, by appending an additional coordinate, initially equal to 1 , then clearing denominators (which is allowed in homogeneous coordinates):

$$
\gamma(t)=\left[2 t^{3}+1: 2 t+t^{4}: t^{2}\right], \quad t \in \mathbb{C P}^{1}
$$

Note that $\gamma(0)=[1: 0: 0]$ and $\gamma(\infty)=[0: 1: 0]$. (To see why the latter expression is correct, rewrite $\gamma(t)$ as $\gamma(1 / s)$, clear denominators, then let $s$ go to 0 .) These are the only two points of $\mathbb{C P}^{1}$ that $\gamma$ sends to $L_{\infty}$.

$\mathcal{D}$ will denote the image of $\gamma$ in either $\mathbb{C}^{2}$ or $\mathbb{C P}^{2}$, and $\mathcal{D}_{\mathbb{E}^{2}}=\mathcal{D} \cap \mathbb{E}^{2}$ is the real deltoid.

In $\mathbb{C}^{2}$ we have

$$
\gamma^{\prime}(t)=\left(2-\frac{2}{t^{3}},-\frac{2}{t^{2}}+2 t\right)=2\left(1-\frac{1}{t^{3}}\right)(1, t)
$$

and so $\gamma^{\prime}(t)$ vanishes precisely when $t$ equals $1, \omega=e^{i 2 \pi / 3}$, or $\omega^{2}=e^{i 4 \pi / 3}$; these cube roots of unity give rise to the three cusps of $\mathcal{D}$. At every other point of $\mathcal{D}$, a tangent vector is $(1, t)$. An equation for the line tangent to $\mathcal{D}$ at $\gamma(t)$ is therefore

$$
\left|\begin{array}{cc}
1 & x-2 t-t^{-2} \\
t & y-2 t^{-1}-t^{2}
\end{array}\right|=0
$$

which is equivalent to

$$
t^{3}-t^{2} x+t y-1=0 .
$$

This equation works equally well at the cusps, where $t^{3}=1$ and (2) reduces to $y=t x$, so each cusp also has a well-defined tangent line, which passes through the origin.

It is worth remarking that in 10 the study of the real deltoid begins, not with any classical construction, but with equation (2), restricted to $y=\bar{x}$ and $|t|=1$, which is simply called the "line equation" of the deltoid.

Equation (2) shows that a generic point $(x, y)$ of $\mathbb{C}^{2}$ lies on three tangent lines of $\mathcal{D}$. A point belongs to $\mathcal{D}$ if and only if at least two of these tangent lines coincide, which is to say that the discriminant of the left side of (21) (as a polynomial in $t$ ) is zero. Thus we obtain an affine equation for $\mathcal{D}$ (and an additional reason to name this set $\mathcal{D}$, since it is where a discriminant vanishes):

$$
x^{2} y^{2}-4\left(x^{3}+y^{3}\right)+18 x y-27=0 .
$$


Now we can also parametrize the dual curve $\mathcal{D}^{\vee}$ in the dual projective plane $\left(\mathbb{C P}^{2}\right)^{\vee}$. From (2), we get the following parametrization of $\mathcal{D}^{\vee}$ :

$$
\check{\gamma}(t)=\left[-t^{2}: t: t^{3}-1\right]^{\vee} .
$$

In particular we see that $\check{\gamma}(0)=\check{\gamma}(\infty)=[0: 0: 1]^{\vee}$, so that the line at infinity in $\mathbb{C P}^{2}$ is tangent to $\mathcal{D}$ at both $\gamma(0)$ and $\gamma(\infty)$. (This tangency can also be seen, less directly, from the fact that $L_{\infty}$ intersects $\mathcal{D}$, a curve of degree 4 , in only two points.) From (4), we can deduce that an equation for $\mathcal{D}^{\vee}$ is

$$
a^{3}+b^{3}=a b c
$$

(when $a, b$, and $c$ are real, this equation produces the folium of Descartes). This curve is smooth except for a self-intersection at $[0: 0: 1]^{\vee}$, which shows that the line at infinity is the only bitangent of $\mathcal{D}$.

Because equation (3) has degree four, a generic line in $\mathbb{C P}^{2}$ will intersect $\mathcal{D}$ in four points. Meanwhile, a generic element of $\mathcal{D}^{\vee}$ (that is, a line tangent to $\mathcal{D}$ ) will intersect $\mathcal{D}$ at two points besides the point of tangency. These other two points of intersection are connected with several interesting geometric properties; we state three of them here for later use. All three have easy algebraic proofs, which we leave to the reader. They are illustrated in Figure 2,
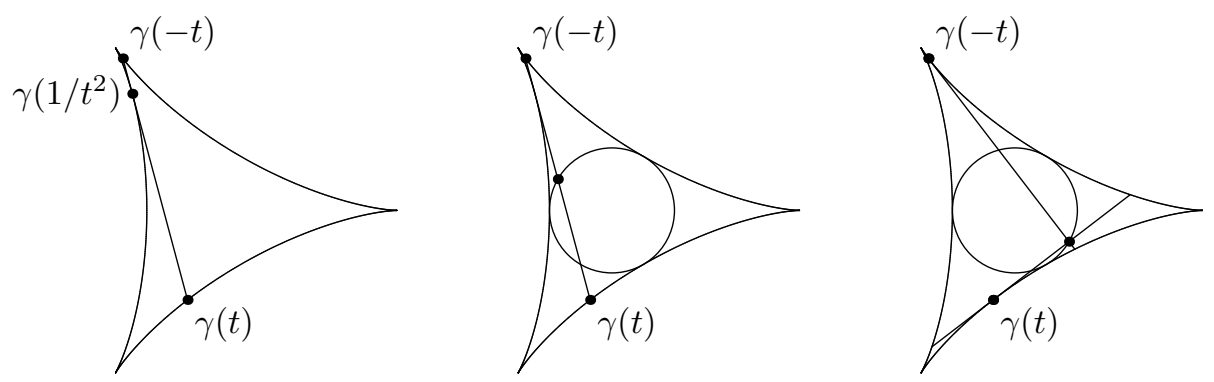

FiguRE 2. Three properties of lines tangent to $\mathcal{D}$.

(A) For all $t \in \mathbb{C} \backslash\{0\}$, the line containing $\gamma(t)$ and $\gamma(-t)$ is tangent to $\mathcal{D}$ at $\gamma\left(1 / t^{2}\right)$.

(B) The midpoint of $\gamma(t)$ and $\gamma(-t)$ in $\mathbb{C}^{2}$ lies on the curve $x y=1$.

(C) The tangent lines $\check{\gamma}(t)$ and $\check{\gamma}(-t)$ intersect at a point also on $x y=1$.

Property (A) will later form the basis for our geometrically-defined dynamical system. Properties (B) and (C) will relate to the critical points of the map.

The curve $\mathcal{C}$ with equation $x y=1$ is, projectively speaking, a conic section. Its intersection $\mathcal{C}_{\mathbb{E}^{2}}$ with the plane $\mathbb{E}^{2}$ is the unit circle, having equation $|x|^{2}=1$.

The real deltoid $\mathcal{D}_{\mathbb{E}^{2}}$ is a Jordan curve in $\mathbb{E}^{2}$; let $K$ be the union of $\mathcal{D}_{\mathbb{E}^{2}}$ with its interior. $K$ consists of those points $x$ such that all solutions to $t^{3}-x t^{2}+\bar{x} t-1=0$ lie on the unit circle $|t|=1$; in other words, these are the points that lie on three "real" tangent lines. (See Figure 3, left and middle.)

\section{The Deltoid MAP.}

In this section we use the geometric properties of $\mathcal{D}$ to define a map $f$ from $\mathbb{C P}^{2}$ to itself. First, we define a natural map $\check{f}$ on the dual curve $\mathcal{D}^{\vee}$. Given $L=T_{\mathbf{x}} \mathcal{D} \in \mathcal{D}^{\vee}$, let $\check{f}(L)$ be 

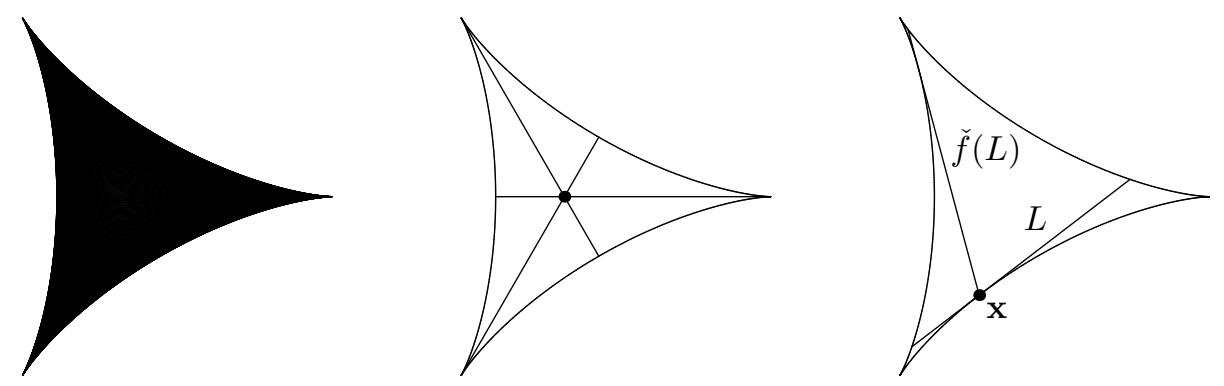

Figure 3. Left: The set $K \subset \mathbb{E}^{2}$ bounded by $\mathcal{D} \cap \mathbb{E}^{2}$. Middle: Tangent lines through the three cusps of $\mathcal{D}$ and their point of intersection at the origin. RIght: For a generic tangent line $L \in \mathcal{D}^{\vee}$ there is another line $\check{f}(L) \in \mathcal{D}^{\vee}$ such that $\check{f}(L)$ is secant to $\mathcal{D}$ at the point $\mathbf{x}$ where $L$ is tangent.

the unique element of $\mathcal{D}^{\vee}$ such that $\{L, \check{f}(L)\}$ is the full set of tangent lines to $\mathcal{D}$ passing through $\mathbf{x}$, as illustrated in Figure 3, right. (Note that $\check{f}(L)$ is the same as $L$ when $\mathbf{x}=\gamma(t)$ for $t \in\left\{1, \omega, \omega^{2}, 0, \infty\right\}$, and it is distinct otherwise.) It follows from property (A) in the previous section that

$$
\check{f}(\check{\gamma}(t))=\check{\gamma}\left(1 / t^{2}\right) \quad \text { for all } t \in \mathbb{C P}^{1} .
$$

In particular, $\check{f}$ fixes $L_{\infty}$ as an element of $\mathcal{D}^{\vee}$, but it is helpful to think of it as exchanging the points of tangency, namely $\gamma(0)$ and $\gamma(\infty)$.

Now we turn to our promised self-map of $\mathbb{C}^{2}$. First we observe that, given $(x, y) \in \mathbb{C}^{2}$, the solutions $t_{1}, t_{2}, t_{3}$ to (2) satisfy $t_{1} t_{2} t_{3}=1$ and

$$
x=t_{1}+t_{2}+t_{3}, \quad y=\frac{1}{t_{1}}+\frac{1}{t_{2}}+\frac{1}{t_{3}} .
$$

Conversely, if $t_{1}, t_{2}, t_{3}$ are chosen to satisfy $t_{1} t_{2} t_{3}=1$, then the formulas (6) provide coefficients for the equation (2) to be solved by $t_{1}, t_{2}, t_{3}$.

Proposition 1. Suppose $\check{\gamma}\left(t_{1}\right), \check{\gamma}\left(t_{2}\right)$, and $\check{\gamma}\left(t_{3}\right)$ are concurrent. Then so are $\check{f}\left(\check{\gamma}\left(t_{1}\right)\right)$, $\check{f}\left(\check{\gamma}\left(t_{2}\right)\right)$, and $\check{f}\left(\check{\gamma}\left(t_{3}\right)\right)$.

Proof. If the point of concurrency lies on $L_{\infty}$, then the result is trivial, as at most two lines are involved. Otherwise a necessary and sufficient condition for concurrency is $t_{1} t_{2} t_{3}=1$. But if $t_{1}, t_{2}$, and $t_{3}$ satisfy this equality, then also $\left(1 / t_{1}^{2}\right)\left(1 / t_{2}^{2}\right)\left(1 / t_{3}^{2}\right)=\left(1 / t_{1} t_{2} t_{3}\right)^{2}=1$.

This proposition provides the basis for defining a map on all of $\mathbb{C P}^{2}$ : given $\mathbf{x} \in \mathbb{C P}^{2}$, let $L_{1}, L_{2}$, and $L_{3}$ be the three elements of $\mathcal{D}^{\vee}$ passing through $\mathbf{x}$ (some of these may coincide). Then define $f(\mathbf{x})$ to be the point at which $\breve{f}\left(L_{1}\right), \breve{f}\left(L_{2}\right)$, and $\check{f}\left(L_{3}\right)$ are concurrent. (See Figure 4.) To handle the special cases of when all three lines $L_{1}, L_{2}$, and $L_{3}$ coincide, we extend by continuity and define $f([1: 0: 0])=[0: 1: 0], f([0: 1: 0])=[1: 0: 0])$, and whenever $\check{f}\left(L_{1}\right)=\check{f}\left(L_{2}\right)=\check{f}\left(L_{3}\right)$ passes through a cusp of $\mathcal{D}, f(\mathbf{x})$ is defined to be that cusp.

With this geometric definition in hand, we find polynomials that describe $f$.

Proposition 2. On $\mathbb{C}^{2}, f$ takes the form $(x, y) \mapsto\left(y^{2}-2 x, x^{2}-2 y\right)$. On $\mathbb{C P}^{2}$, this extends to $[x: y: z] \mapsto\left[y^{2}-2 x z: x^{2}-2 y z: z^{2}\right]$. On $L_{\infty}, f$ has the form $\zeta \mapsto 1 / \zeta^{2}$. 

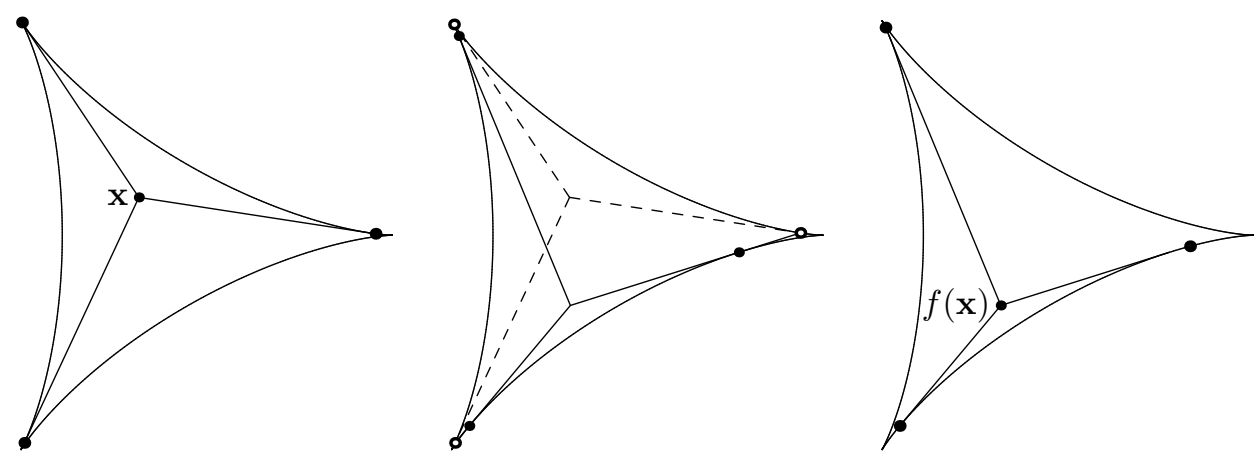

Figure 4. Geometric definition of $f$. Any point $\mathbf{x} \in \mathbb{C P}^{2}$ lies on three tangent lines of $\mathcal{D}$ (counted with multiplicity). The point of tangency for each of these lines lies on another element of $\mathcal{D}^{\vee}$, as seen in Figure 3. The resulting collection of three new tangent lines (again, counted with multiplicity) is concurrent at $f(\mathbf{x})$.

Proof. If $(x, y) \in \mathbb{C}^{2}$, and $t_{1}, t_{2}$, and $t_{3}$ are the roots of (2), then by the observations surrounding equation (6) , we have

$$
f(x, y)=\left(\frac{1}{t_{1}^{2}}+\frac{1}{t_{2}{ }^{2}}+\frac{1}{t_{3}^{2}}, t_{1}^{2}+t_{2}^{2}+t_{3}^{2}\right) .
$$

Now we observe that

$$
\left(\frac{1}{t_{1}}+\frac{1}{t_{2}}+\frac{1}{t_{3}}\right)^{2}-2\left(\frac{1}{t_{1} t_{2}}+\frac{1}{t_{2} t_{3}}+\frac{1}{t_{3} t_{1}}\right)=\frac{1}{t_{1}{ }^{2}}+\frac{1}{t_{2}{ }^{2}}+\frac{1}{t_{3}{ }^{2}}
$$

and

$$
\left(t_{1}+t_{2}+t_{3}\right)^{2}-2\left(t_{1} t_{2}+t_{2} t_{3}+t_{3} t_{1}\right)=t_{1}{ }^{2}+t_{2}{ }^{2}+t_{3}{ }^{2},
$$

which proves the result on $\mathbb{C}^{2}$. The formula on $\mathbb{C P}^{2}$ is then obtained by a standard homogenization process. Because $L_{\infty}$ is defined by $z=0$, on this line the map becomes $[x: y: 0] \mapsto\left[y^{2}: x^{2}: 0\right]$; if we set $\zeta=y / x$, the result for $L_{\infty}$ becomes clear. Alternatively, for $L_{\infty}$ we could use the observations made previously that $f(\check{\gamma}(t))=\check{\gamma}\left(1 / t^{2}\right)$ and that $\check{\gamma}(t)$ intersects $L_{\infty}$ at $[1: t: 0]$, so $\zeta=t$.

\section{Julia set, Fatou Set, and Green function.}

Having defined the deltoid map $f$, we turn to some of its dynamical properties. Ideally, for any point $\mathbf{x} \in \mathbb{C P}^{2}$, we would like to be able to predict the behavior of its orbit under $f$, which is the sequence $\mathbf{x}, f(\mathbf{x}), f^{2}(\mathbf{x}), f^{3}(\mathbf{x}), \ldots$, and also to say something about the orbits of points near $\mathbf{x}$. (Here and in the rest of the article $f^{n}$ denotes the composition of $f$ with itself $n$ times; this notation is standard in dynamical systems.) From the construction of $f$, we can already see that it has some exceptional properties: the deltoid $\mathcal{D}$ is forward invariant, meaning $f(\mathcal{D})=\mathcal{D}$, and $f$ also sends each line tangent to $\mathcal{D}$ to another such line. These tangent lines will continue to be key in studying properties of $f$.

Notice that $f$ commutes with the involution $\iota(x, y)=(y, x)$. The composition $\iota \circ f=f \circ \iota$ is studied by Uchimura in [15, 16, 17] and Nakane in [11]. The dynamical properties of $f$ and $\iota \circ f$ are essentially identical. 
A fundamental tool in complex dynamics is the partition of the dynamical space into the Fatou set, where the dynamics are "simple," and the Julia set, where the dynamics are "chaotic." More precisely, the Fatou set $\Omega=\Omega_{f}$ is the largest open set of $\mathbb{C P}^{2}$ on which the iterates of $f$ locally form an equicontinuous family; thus if $\mathbf{x}$ and $\mathbf{y}$ are points of $\Omega$ that are sufficiently near each other, then $f^{n}(\mathbf{x})$ and $f^{n}(\mathbf{y})$ remain close (in $\left.\mathbb{C P}^{2}\right)$ as $n$ increases. The Julia set $J=J_{f}$ is the complement of $\Omega$; thus if $\mathbf{x}$ is in $J$ and $\mathbf{y}$ is close to $\mathbf{x}$, then $f^{n}(\mathbf{x})$ and $f^{n}(\mathbf{y})$ may be very far apart.

On $L_{\infty}$, as we have seen, $f$ reduces to the power map $\zeta \mapsto 1 / \zeta^{2}$. This map of $\mathbb{C P}^{1}$ exchanges 0 and $\infty$ (in $\mathbb{C P}^{2}$, these are the points $[1: 0: 0]$ and $[0: 1: 0]$ ), and so these two points form a period 2 orbit. If $|\zeta| \neq 1$, then $\zeta^{(-2)^{n}}$ approaches the previously observed period 2 orbit. If $|\zeta|=1$, then $\zeta^{(-2)^{n}}$ remains on the unit circle, while some nearby points are drawn to the $\{0, \infty\}$ orbit. Thus the Julia set of $f$ on $L_{\infty}$ is the circle at infinity, and the Fatou set in $L_{\infty}$ has two components, one containing 0 and the other $\infty$.

To determine the Julia and Fatou sets of $f$ in $\mathbb{C}^{2}$, we introduce the Green function $G=G_{f}$ of $f$, which is defined [3, 8] by

$$
G(\mathbf{x})=\lim _{n \rightarrow \infty} \frac{1}{2^{n}} \log ^{+}\left\|f^{n}(\mathbf{x})\right\|,
$$

where $\log ^{+}=\max \{\log , 0\}$, and $\|\cdot\|$ is any norm on $\mathbb{C}^{2}$. This function measures how quickly points of $\mathbb{C}^{2}$ escape to infinity under iteration of $f$; it is zero precisely for those points whose orbits are bounded, which comprise the set $K$. It is a continuous, subharmonic function on $\mathbb{C}^{2}$, and it satisfies the functional equation $G(f(x, y))=2 G(x, y)$.

For most self-maps of $\mathbb{C}^{2}$, the Green function cannot be explicitly calculated. The deltoid map is an exception.

Proposition 3. The Green function $G$ of the deltoid map $f$ can be calculated as follows: given $(x, y) \in \mathbb{C}^{2}$, let $t_{1}, t_{2}$, and $t_{3}$ be the solutions to (2). Then

$$
G(x, y)=\log \max \left\{\left|t_{1}\right|,\left|t_{2}\right|,\left|t_{3}\right|, \frac{1}{\left|t_{1}\right|}, \frac{1}{\left|t_{2}\right|}, \frac{1}{\left|t_{3}\right|}\right\} .
$$

Notice that we do not need to use $\log ^{+}$in (7), because the set over which the maximum is taken contains at least one element that is greater than or equal to 1.

Proof of Proposition [3. Using the $L^{\infty}$ norm on $\mathbb{C}^{2}$, we have

$$
G(x, y)=\lim _{n \rightarrow \infty} \frac{1}{2^{n}} \log ^{+} \max \left\{\left|t_{1}{ }^{2^{n}}+t_{2} 2^{n}+t_{3}{ }^{n}\right|,\left|\frac{1}{t_{1}^{2^{n}}}+\frac{1}{t_{2} 2^{n}}+\frac{1}{t_{3} 2^{n}}\right|\right\} \text {. }
$$

Set $\tau=\max \left\{\left|t_{1}\right|,\left|t_{2}\right|,\left|t_{3}\right|,\left|t_{1}\right|^{-1},\left|t_{2}\right|^{-1},\left|t_{3}\right|^{-1}\right\}$. Then $\tau \geq 1$, and we have

$$
\begin{aligned}
& \frac{1}{2^{n}} \log \max \left\{\left|t_{1}^{2^{n}}+t_{2}^{2^{n}}+t_{3}^{2^{n}}\right|,\left|\frac{1}{t_{1}^{2^{n}}}+\frac{1}{t_{2}^{2^{n}}}+\frac{1}{t_{3}^{2^{n}}}\right|\right\}-\log \tau \\
& =\frac{1}{2^{n}} \log \max \left\{\frac{\left|t_{1}^{2^{n}}+t_{2}^{2^{n}}+t_{3}^{2^{n}}\right|}{\tau^{2^{n}}}, \frac{1}{\tau^{2^{n}}}\left|\frac{1}{t_{1}^{2^{n}}}+\frac{1}{t_{2} 2^{n}}+\frac{1}{t_{3}{ }^{n}}\right|\right\} .
\end{aligned}
$$

By our choice of $\tau$, the maximum of the set in (9) is bounded by 3 . Therefore, as $n$ tends to $\infty$, the difference in (8) tends to 0 . This shows that $G(x, y)=\log \tau$, as claimed. 
In terms of the Green function, $\Omega$ is the set of points where $d d^{c} G$ vanishes. Here $d d^{c}=$ $\frac{i}{2 \pi} \partial \bar{\partial}$ is the so-called pluri-Laplacian, and the derivatives should properly be interpreted as currents ("differential forms with distributional coefficients"); for us, however, it is sufficient to know where $d d^{c} G=0$. Because $d d^{c} \log ^{+}|t|$ vanishes except on the unit circle $S^{1}$, we obtain the following characterization of $J$.

Proposition 4. The Julia set of $f$ is the set $J$ of points $[x: y: z] \in \mathbb{C P}^{2}$ such that the polynomial $z\left(t^{3}-1\right)-x t^{2}+y t$ has at least one root on $S^{1}$.

Given our geometric definition of $f$, this result is not surprising: as we have seen, the line $\check{\gamma}(t) \in \mathcal{D}^{\vee}$ intersects $L_{\infty}$ at $[1: t: 0]$, and the circle at infinity, where $|t|=1$, is precisely the Julia set of $\left.f\right|_{L_{\infty}}$.

Nakane [11] provided a description of the foliation of $J$ by "stable disks" of the circle at infinity, as well as how external rays land at points of $K$. We shall take a different perspective and consider the intersection of $J$ with complex lines in $\mathbb{C}^{2}$ parallel to the $x$ - and $y$-axes. In order to describe the result, however, we must invoke some classical differential geometry.

Given a curve $C$ and a point $O$ in $\mathbb{E}^{2}$, the pedal curve of $C$ with respect to $O$ is the locus of points $P$ such that $P$ is the orthogonal projection of $O$ onto a line tangent to $C$. (See Figure 5 for some examples.)
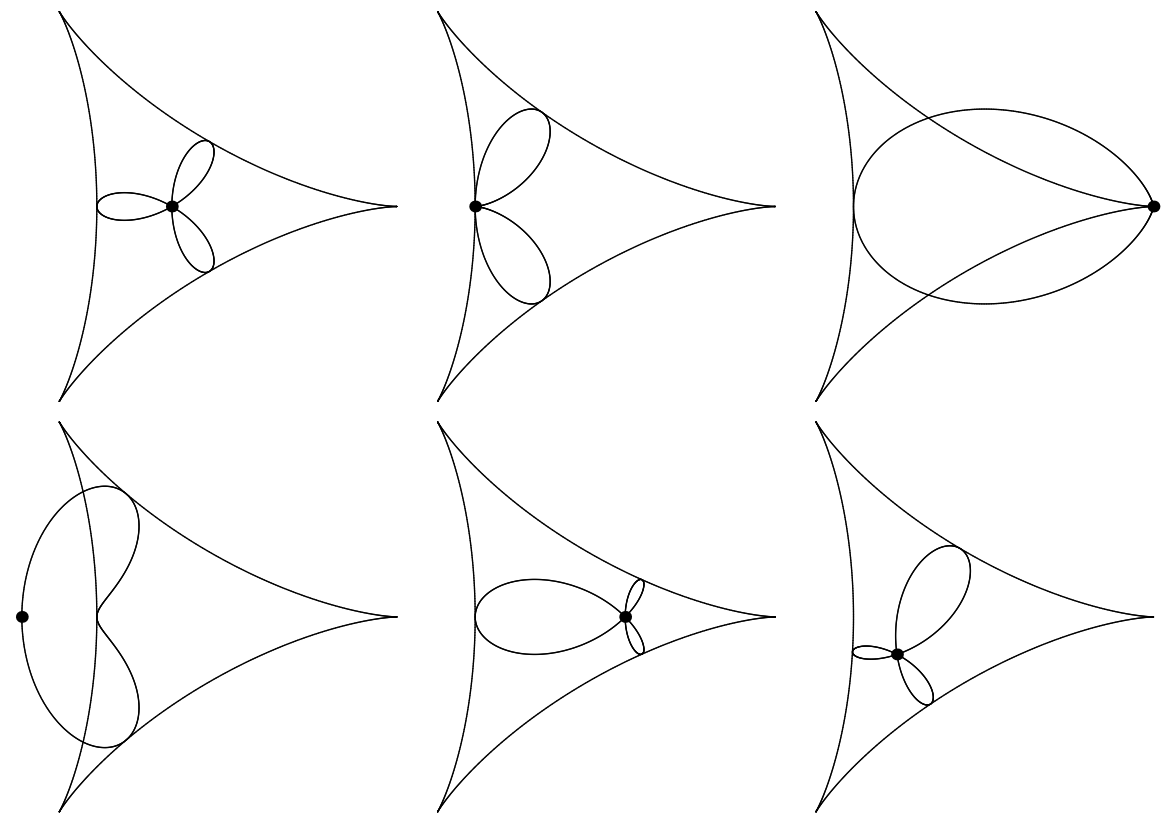

Figure 5. Some pedal curves of the real deltoid in $\mathbb{E}^{2}$. In each image the point $O$ is indicated by a dot. Top: With respect to the center (a trifolium), with respect to the point opposite a cusp (a bifolium), and with respect to a cusp (a simple folium). Bоттом: With respect to an exterior point, with respect to an interior point on an axis of symmetry, and with respect to a generic interior point.

At this point we can state our first main result, which says that the Julia set of the deltoid map on $\mathbb{C}^{2}$ geometrically decomposes into a disjoint union of pedal curves of the real deltoid. 
Theorem 1. The intersection of $J$ with a line $L \neq L_{\infty}$ through $[1: 0: 0]$ (that is, parallel to the $x$-axis in $\mathbb{C}^{2}$ ) is the pedal curve of the real deltoid with respect to the $x$-coordinate of $L \cap \mathbb{E}^{2}$. Likewise, the intersection of $J$ with a line parallel to the $y$-axis is the pedal curve of the real deltoid with respect to the $y$-coordinate of the intersection of this line and $\mathbb{E}^{2}$.

To prove this result, we will use the following projection from $\mathbb{C}^{2}$ to $\mathbb{E}^{2}$ :

$$
\operatorname{pr}_{\mathbb{E}^{2}}(x, y)=\left(\frac{x+\bar{y}}{2}, \frac{y+\bar{x}}{2}\right) \text {. }
$$

This projection is orthogonal with respect to the standard Hermitian inner product on $\mathbb{C}^{2}$, namely $\left(x_{1}, y_{1}\right) \cdot\left(x_{2}, y_{2}\right)=x_{1} \overline{x_{2}}+y_{1} \overline{y_{2}}$. Conveniently, it also preserves each complex line that is tangent to $\mathcal{D}$ at a point of $\mathcal{D}_{\mathbb{E}^{2}}$, which is the content of the next lemma.

Lemma 1. If $|t|=1$ and $(x, y) \in \check{\gamma}(t)$, then also $\operatorname{pr}_{\mathbb{E}^{2}}(x, y) \in \check{\gamma}(t)$.

Proof. By assumption, $t$ and $(x, y)$ satisfy equation (2) $t^{3}-t^{2} x+t y-1=0$, as well as its conjugate $\bar{t}^{3}-\bar{t}^{2} \bar{x}+\bar{t} \bar{y}-1=0$. Because $|t|=1$, we have $\bar{t}=t^{-1}$, and so, after multiplying the conjugate of (2) by $t^{3}$ we obtain $1-t \bar{x}+t^{2} \bar{y}-t^{3}=0$. Subtracting this latter equation from (2) and dividing by 2 produces

$$
t^{3}-t^{2}\left(\frac{x+\bar{y}}{2}\right)+t\left(\frac{y+\bar{x}}{2}\right)-1=0
$$

as desired.

A line in $\mathbb{C}^{2}$ parallel to the $x$-axis is determined by its $y$-coordinate. Let $L_{\alpha}$ be the line with equation $y=\bar{\alpha}$. The intersection of $L_{\alpha}$ with $\mathbb{E}^{2}$ is

$$
L_{\alpha} \cap \mathbb{E}^{2}=\{(\alpha, \bar{\alpha})\} .
$$

The restriction of $\operatorname{pr}_{\mathbb{E}^{2}}$ to $L_{\alpha}$ is a bijection, whose inverse $\lambda_{\alpha}: \mathbb{E}^{2} \rightarrow L_{\alpha}$ is the affine map

$$
\lambda_{\alpha}(x, \bar{x})=(2 x-\alpha, \bar{\alpha}) .
$$

Notice, however, that with respect to the metrics induced on $\mathbb{E}^{2}$ and $L_{\alpha}$ by the Hermitian inner product on $\mathbb{C}^{2}, \lambda_{\alpha}$ is not just affine, but a similarity. To prove Theorem 1 , therefore, it suffices to show that $\operatorname{pr}_{\mathbb{E}^{2}}\left(J \cap L_{\alpha}\right)$ is the pedal curve of $\mathcal{D} \cap \mathbb{E}^{2}$ with respect to $(\alpha, \bar{\alpha})$. Or what is the same, we need to show that for all $t \in S^{1}$, the point $(x, \bar{x}) \in \mathbb{E}^{2}$ is the orthogonal projection of $(\alpha, \bar{\alpha})$ onto $\check{\gamma}(t) \cap \mathbb{E}^{2}$ if and only if $\lambda_{\alpha}(x, \bar{x})$ is in $\check{\gamma}(t)$.

If $|t|=1$, then the Hermitian inner product of the vectors $(1, t)$ and $(1,-t)$ is zero, so any two lines in $\mathbb{C}^{2}$ of the form $y=t x+b_{1}$ and $y=-t x+b_{2}$ are orthogonal.

Proof of Theorem 1 . Let $|t|=1$. The intersection of $\check{\gamma}(t)$ and $\mathbb{E}^{2}$ has the equation

$$
t^{3}-t^{2} x+t \bar{x}-1=0, \quad \text { or } \quad \bar{x}=t x-t^{2}+t^{-1} .
$$

The line through $(\alpha, \bar{\alpha})$ that is orthogonal to $\check{\gamma}(t)$ is therefore

$$
\bar{x}-\bar{\alpha}=-t(x-\alpha) \text {. }
$$

These latter two equations together imply (by eliminating $\bar{x}$ ) that

$$
t x-t^{2}+t^{-1}=\bar{\alpha}-t(x-\alpha),
$$

and solving for $x$ produces

$$
x=\frac{1}{2}\left(\alpha+t+\bar{\alpha} t^{-1}-t^{-2}\right) .
$$


On the other hand, if $\lambda_{\alpha}(x, \bar{x}) \in J$, then

$$
t^{3}-t^{2}(2 x-\alpha)+t \bar{\alpha}-1=0
$$

which produces the same solution for $x$, as desired.

The proof for the intersection of $J$ with a line parallel to the $y$-axis is virtually identical.

From this geometric description of the intersection of $J$ with a horizontal or vertical line, we can find an algebraic equation for $J$ in $\mathbb{C}^{2}$.

Corollary 1. The Julia set of $f$ is the real hypersurface in $\mathbb{C}^{2}$ having the equation

$$
2 \operatorname{Re}(x-\bar{y})^{3}+\operatorname{Re}(x-\bar{y})^{2}\left(\bar{x}^{2}-y^{2}\right)=0 .
$$

Proof. Start in $\mathbb{E}^{2}$ with the real lines

$$
t^{3}-t^{2} x+t \bar{x}-1=0 \quad \text { and } \quad \bar{x}-\bar{\alpha}=-t(x-\alpha),
$$

then eliminate $t$ to get

$$
\left(\frac{\bar{x}-\bar{\alpha}}{x-\alpha}\right)^{3}+\left(\frac{\bar{x}-\bar{\alpha}}{x-\alpha}\right)^{2} x+\left(\frac{\bar{x}-\bar{\alpha}}{x-\alpha}\right) \bar{x}+1=0
$$

Now a point $(x, y) \in \mathbb{C}^{2}$ is in $J$ if $\operatorname{pr}_{\mathbb{E}^{2}}(x, y)$ satisfies this equation (meaning we replace $x$ with $(x+\bar{y}) / 2$ and $\bar{x}$ with $(\bar{x}+y) / 2)$ when $\alpha=\bar{y}$, which yields

$$
2(\bar{x}-y)^{3}+(\bar{x}-y)^{2}\left(x^{2}-\bar{y}^{2}\right)+(x-\bar{y})^{2}\left(\bar{x}^{2}-y^{2}\right)+2(x-\bar{y})^{3}=0 .
$$

This is equivalent to the desired equation.

Note that in particular the equation in Corollary 1 is satisfied when $y=\bar{x}$, so $\mathbb{E}^{2}$ is entirely contained in $J$. This is to be expected, because every point of $\mathbb{E}^{2}$ lies on a line that intersects $L_{\infty}$ on the circle at infinity.

To end this section, we provide a description of the Fatou set $\Omega$.

Corollary 2. $\Omega$ has two components, each of which is biholomorphic to $(\mathbb{D} \times \mathbb{D}) / \sigma$, where $\mathbb{D}$ is the open unit disk in $\mathbb{C}$ and $\sigma$ is the involution $\sigma(u, v)=(v, u)$. These two components are exchanged by $f$.

Proof of Corollary Q Define the following two functions from $\mathbb{C}^{2}$ to $\mathbb{C P}^{2}$ :

$$
\begin{aligned}
& \Psi_{x}(u, v)=\left[u^{2} v+u v^{2}+1: u+v+u^{2} v^{2}: u v\right], \\
& \Psi_{y}(u, v)=\left[u+v+u^{2} v^{2}: u^{2} v+u v^{2}+1: u v\right] .
\end{aligned}
$$

Direct computation shows that

$$
\left(f \circ \Psi_{x}\right)(u, v)=\Psi_{y}\left(u^{2}, v^{2}\right) \quad \text { and } \quad\left(f \circ \Psi_{y}\right)(u, v)=\Psi_{x}\left(u^{2}, v^{2}\right),
$$

and for $u v \neq 0, \Psi_{x}(1 / u, 1 / v)=\Psi_{y}(u, v)$. Geometrically, $u$ and $v$ are the $t$-parameters for two of the lines in $\mathcal{D}^{\vee}$ passing through $\Psi_{x}(u, v)$, the third being $1 / u v$. Thus, $\Psi_{x}(u, v)$ is contained in $J$ if and only if either $u$ or $v$ lies on the unit circle, and the same holds for $\Psi_{y}(u, v)$. Together, $\Psi_{x}$ and $\Psi_{y}$ cover all of $\mathbb{C P}^{2}$.

By definition of $J$ as the complement of $\Omega$, we see that $\Omega$ is covered by the two images of $\mathbb{D} \times \mathbb{D}$ via $\Psi_{x}$ and $\Psi_{y}$. Thus $\Omega$ has two connected components. The polynomials defining $\Psi_{x}$ and $\Psi_{y}$ are symmetric in $u$ and $v$, and distinct unordered pairs $\{u, v\}$ lead to different points of $\mathbb{C P}^{2}$ by $\Psi_{x}$ and $\Psi_{y}$. This proves the result. 
The functions $\Psi_{x}$ and $\Psi_{y}$ are variants of the function $\Psi$ used in [11] as an "inverse Böttcher coordinate" on the Julia set of $f$. We can see from the formulas for $f \circ \Psi_{x}$ and $f \circ \Psi_{y}$ how the orbit of any point of $\Omega$ tends uniformly and super-exponentially to the orbit consisting of $\Psi_{x}(0,0)=[1: 0: 0]$ and $\Psi_{y}(0,0)=[0: 1: 0]$.

\section{ITERATED MONODROMY GROUP OF THE DELTOID MAP.}

We begin this final section with one more exceptional property of $f$.

The Jacobian determinant of $f$ at $(x, y) \in \mathbb{C}^{2}$ is $4(1-x y)$. Thus the locus of critical points in $\mathbb{C}^{2}$ is the curve $\mathcal{C}$ having equation $x y=1$, whose importance was previously noted in Section 3. Indeed, because the lines $\check{\gamma}(t)$ and $\check{\gamma}(-t)$ have the same image under $\check{f}$, their point of intersection must be a critical point of $f$; by property $(\mathrm{C})$, all such points lie on $\mathcal{C}$.

If we parametrize $\mathcal{C}$ by $(t, 1 / t)$, then we find that the image of a point of $\mathcal{C}$ can be written as

$$
f\left(t, \frac{1}{t}\right)=\left(-2 t+\frac{1}{t^{2}}, t^{2}-\frac{2}{t}\right)=\gamma(-t),
$$

and so we see that $f(\mathcal{C})=\mathcal{D}$. Because $\mathcal{D}$ is forward invariant under $f$, we conclude that $f$ is post-critically finite, meaning that the post-critical locus $\bigcup_{n \geq 1} f^{n}(\mathcal{C})$ is an algebraic curvein this case, $\mathcal{D}$ itself. (Post-critically finite maps of $\mathbb{C P}^{2}$ were introduced in [5], under the name of "critically finite rational maps.")

Set $\mathcal{X}=\mathbb{C}^{2} \backslash \mathcal{D}$ and $\mathcal{X}_{1}=\mathcal{X} \backslash \mathcal{C}$. Then the above property implies that $\left.f\right|_{\mathcal{X}_{1}}$ is a covering map from $\mathcal{X}_{1}$ to $\mathcal{X}$, called a partial self-covering of $\mathcal{X}$. Let $\mathbf{x}_{0}=(0,0) \in \mathcal{X}$; then the fundamental group $\pi_{1}\left(\mathcal{X}, \mathbf{x}_{0}\right)$ permutes the set of preimages of $\mathbf{x}_{0}$ by $f$ in a standard way: given $[\eta] \in \pi_{1}\left(\mathcal{X}, \mathbf{x}_{0}\right)$ and $\mathbf{y} \in f^{-1}\left(\mathbf{x}_{0}\right)$, use $f$ to lift $\eta$ to a path $\bar{\eta}$ starting at $\mathbf{y}$, and let $[\eta] \cdot \mathbf{y}$ be the endpoint of $\bar{\eta}$. This defines a homomorphism $\mu_{f}$ from $\pi_{1}\left(\mathcal{X}, \mathbf{x}_{0}\right)$ to the symmetric group on $f^{-1}\left(\mathbf{x}_{0}\right)$, called the monodromy homomorphism.

Likewise, if we set $\mathcal{X}_{n}=f^{-n}(\mathcal{X})$, then $\left.f^{n}\right|_{\mathcal{X}_{n}}$ is a covering map, and $\pi_{1}\left(\mathcal{X}, \mathbf{x}_{0}\right)$ acts on $f^{-n}\left(\mathbf{x}_{0}\right)$ by the monodromy homomorphism $\mu_{f^{n}}$. The intersection

$$
\kappa_{f}=\bigcap_{n \geq 1} \operatorname{ker} \mu_{f^{n}}
$$

is a normal subgroup of $\pi_{1}\left(\mathcal{X}, \mathbf{x}_{0}\right)$, consisting of all elements $[\eta]$ such that every lift of $\eta$ by every iterate of $f$ remains a loop. The quotient

$$
\operatorname{IMG}(f)=\pi_{1}\left(\mathcal{X}, \mathbf{x}_{0}\right) / \kappa_{f}
$$

is called the iterated monodromy group of $f$. (See [6, 12] for details.)

Iterated monodromy groups are a relatively recent addition to the complex dynamics toolbox. They have already proved useful in classification problems [1] and in determining the shape of Julia sets more complicated than that of the deltoid map [13]. Nevertheless, only a few such groups have been explicitly calculated, especially for maps in dimension greater than 1. A nice feature of $f$ is that $\operatorname{IMG}(f)$ can be found directly from the definition, which is how we will prove our second main result.

Theorem 2. IMG $(f)$ is isomorphic to the affine Coxeter group $\tilde{A}_{2}$. 
$\tilde{A}_{2}$ can be realized geometrically as the group generated by reflections across the sides of an equilateral triangle in the plane. It has the group presentation

$$
\tilde{A}_{2}=\left\langle g_{1}, g_{2}, g_{3} \mid \forall k g_{k}^{2}=1, \forall j \forall k\left(g_{j} g_{k}\right)^{3}=1\right\rangle .
$$

On the other hand, the fundamental group $\pi_{1}\left(\mathcal{X}, \mathbf{x}_{0}\right)$ is isomorphic to the related Artin group

$$
\bar{A}_{2}=\left\langle h_{1}, h_{2}, h_{3} \mid \forall j \forall k h_{j} h_{k} h_{j}=h_{k} h_{j} h_{k}\right\rangle
$$

(see [2] for a proof). Note that in $\tilde{A}_{2}$, the relation $\left(g_{j} g_{k}\right)^{3}=1$ is equivalent to $g_{j} g_{k} g_{j}=g_{k} g_{j} g_{k}$, and so $\tilde{A}_{2}$ can be obtained from $\bar{A}_{2}$ by adding the relations $h_{k}^{2}=1$ for $k=1,2,3$. We will accomplish this in Lemma 2, then show that no additional relations are present in $\operatorname{IMG}(f)$.

First we find a useful set of generators for $\pi_{1}\left(\mathcal{X}, \mathbf{x}_{0}\right)$ : these can be chosen as circles contained in the lines $\check{\gamma}(\omega), \check{\gamma}\left(\omega^{2}\right)$, and $\check{\gamma}(1)$ and passing through $\mathbf{x}_{0}$. To see why, we use the Zariski-van Kampen theorem [18, 22], which states that generators can be obtained by taking a sufficiently general line $L$ and drawing loops around the finite set of points $L \cap \mathcal{D}$. The condition on $L$ is that $L \cap \mathcal{D}$ should have four distinct points in $\mathbb{C}^{2}$. We choose a line of the form $L=\{(x, y) \mid x+y=-a\}$, where $2<a<3$. Then (3) implies that $\gamma(t)$ lies on $L$ if

$$
t^{4}+2 t^{3}+a t^{2}+2 t+1=0 \text {, }
$$

and our choice of $a$ ensures that all solutions of this equation lie on the unit circle, which means all points of intersection in $L \cap \mathcal{D}$ lie in $\mathbb{E}^{2}$. (See Figure 6 , left.) Thus the four points of $L \cap \mathcal{D}$ lie in a straight (real) line, and so we can draw small loops around these inside the (complex) line $L$. Each such loop intersects $\mathbb{E}^{2}$ in two points: one in $K$, and one outside.
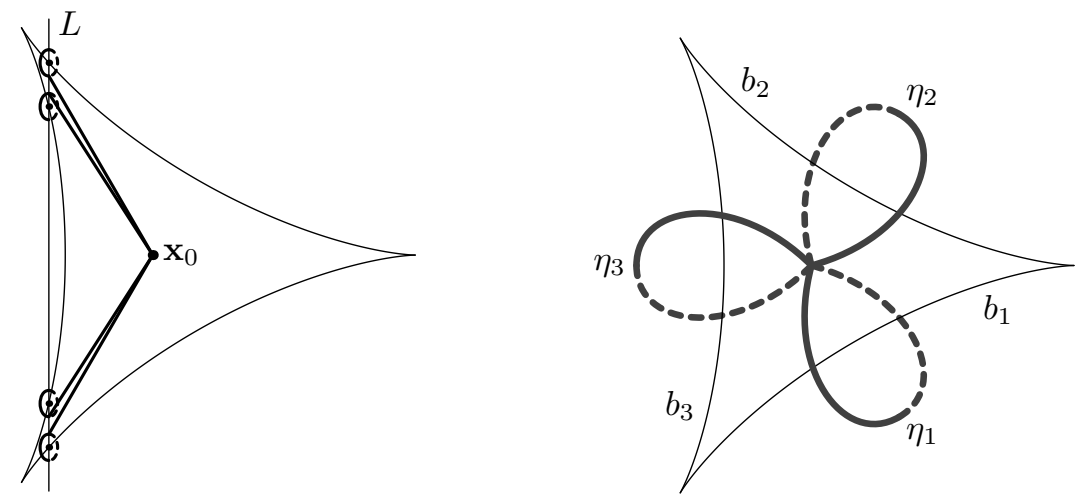

Figure 6. LEFT: The complex line $L$ with equation $x+y=a$ intersects the deltoid $\mathcal{D}$ at four points, all contained in $\mathbb{E}^{2}$, provided $-3<a<-2$. Around each point of intersection, draw a loop inside $L$ that intersects $K$ at one point. When connected to $\mathbf{x}_{0}$ by additional segments in $K$, these loops generate $\pi_{1}\left(\mathcal{X}, \mathbf{x}_{0}\right)$. Right: Generators for $\pi_{1}\left(\mathcal{X}, \mathbf{x}_{0}\right)$, homotopic to those found in left picture. Each loop $\eta_{k}$ is contained in the complex line $\check{\gamma}\left(\omega^{k}\right)$, which intersects $\mathcal{D}$ at the cusp $\gamma\left(\omega^{k}\right)$ and at the midpoint of the opposite branch $b_{k}$.

Connect each loop in $L$ from the point where it intersects $K$ to $\mathbf{x}_{0}$ with a line segment, so that it becomes an element of $\pi_{1}\left(\mathcal{X}, \mathbf{x}_{0}\right)$ (with orientation given by the complex line in which it lies). Let's label these elements. The real deltoid has three cusps, and between these lie three "branches":

- one from $\gamma(1)$ to $\gamma(\omega)$, 
- one from $\gamma(\omega)$ to $\gamma\left(\omega^{2}\right)$, and

- one from $\gamma\left(\omega^{2}\right)$ to $\gamma(1)$.

Call these branches, respectively, $b_{2}, b_{3}$, and $b_{1}$, so that $b_{k}$ and $b_{k+1}$ meet at the cusp to which $\check{\gamma}\left(\omega^{k+2}\right)$ lies tangent. (All indices are computed modulo 3.) The loops in $L$ surrounding $b_{1}$ and $b_{2}$ are homotopic in $\mathcal{X}$ to loops that lie in $\breve{\gamma}(\omega)$ and $\breve{\gamma}\left(\omega^{2}\right)$. On the other hand, the two loops surrounding $b_{3}$ are both homotopic to the same loop in $\check{\gamma}(1)$. Thus $\pi_{1}\left(\mathcal{X}, \mathbf{x}_{0}\right)$ is generated by three elements, which have representatives lying in the lines $\check{\gamma}(\omega), \check{\gamma}\left(\omega^{2}\right)$, and $\check{\gamma}(1)$. Call these, respectively, $\eta_{1}, \eta_{2}$, and $\eta_{3}$, so that $\eta_{k}$ wraps around $b_{k} \cap \check{\gamma}\left(\omega^{k}\right)$. (See Figure 6, right.)

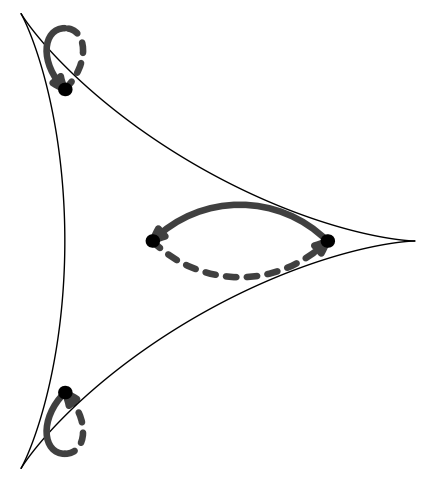

Figure 7 . The four lifts of $\eta_{3}$ by $f$. The loops lie in $\check{\gamma}(-1)$, and the arcs lie in $\check{\gamma}(1)$.

Lemma 2. For each $k=1,2,3$ and for all $n \geq 1, \mu_{f^{n}}\left(\left[\eta_{k}\right]\right)$ has order 2 .

Proof. We want to show that every lift of $\eta_{k}$ by every iterate of $f$ is either a closed loop, or forms a closed loop with one other lift. We will use the fact that every lift of $\eta_{k}$ by any iterate of $f$ is contained in some line $\check{\gamma}(t) \in \mathcal{D}^{\vee}$.

The line $\check{\gamma}(t)$, when $t \in \mathbb{C} \backslash\{0\}$, can be parametrized by

$$
\sigma_{t}(s)=\left(t+\frac{s}{\sqrt{t}}, \frac{1}{t}+s \sqrt{t}\right), \quad s \in \mathbb{C},
$$

as may be checked directly from the equation for $\check{\gamma}(t)$. (Here, $\sqrt{t}$ can be either square root of $t$.) This parametrization of $\gamma(t)$ has the nice feature that when $s=0$, the resulting point lies on the critical locus $\mathcal{C}$, since it is the midpoint of $\gamma(\sqrt{t})$ and $\gamma(-\sqrt{t})$ (see property (B) from Section [3).

Now when we apply $f$ to $\sigma_{t}(s)$, we obtain

$$
f\left(\sigma_{t}(s)\right)=\left(\frac{1}{t^{2}}+\left(s^{2}-2\right) t, t^{2}+\left(s^{2}-2\right) \frac{1}{t}\right)=\sigma_{1 / t^{2}}\left(s^{2}-2\right) .
$$

So we just need to consider the possible lifts of a closed curve in $\mathbb{C}$ by the polynomial $T(s)=s^{2}-2$, avoiding the post-critical set of $T$. (See, for example, Figure 7 , which illustrates the four lifts of $\eta_{3}$ by $f$.)

The critical point of $T(s)$ is 0 , and its critical value is -2 . The image of -2 by $T(s)$ is 2 , which is a fixed point. Let $\eta$ be any loop in $\mathbb{C}$ that does not pass through -2 or 2 . If $\eta$ does not encircle -2 , then it lifts to a pair of disjoint loops; if $\eta$ encircles 2 , then one of these loops 
encircles 2 and one encircles -2 , otherwise neither lift encircles -2 . If $\eta$ does encircle -2 , then it lifts to a double cover of itself, consisting of two arcs, that does not encircle -2 .

In other words, Lemma 2 says that the square of each generator $\left[\eta_{k}\right]$ is in $\kappa_{f}$. Together with the relations in $\pi_{1}\left(\mathcal{X}, \mathbf{x}_{0}\right)=\bar{A}_{2}$, this result implies that $\operatorname{IMG}(f)$ is a quotient of $\tilde{A}_{2}$. To complete the proof of Theorem 2 , we need to show that no additional relations are present in $\operatorname{IMG}(f)$.

Proof of Theorem 2. Recall the realization of $\tilde{A}_{2}$ as the group generated by reflections $\rho_{1}, \rho_{2}$, $\rho_{3}$ across the sides of an equilateral triangle. This group can be expressed as the semidirect product $\Lambda \rtimes D_{3}$, where $\Lambda$ is the normal subgroup consisting of translations (isomorphic to $\mathbb{Z}^{2}$ ) and $D_{3}$ is the subgroup that fixes a vertex of the triangle (the dihedral group of order 6). $D_{3}$ is generated by the reflections in two adjacent sides of the triangle.

Suppose $\phi: \tilde{A}_{2} \rightarrow \operatorname{IMG}(f)$ is the homomomorphism that sends $\rho_{k}$ to $\left[\eta_{k}\right] \kappa_{f}$. If $\operatorname{ker} \phi \cap D_{3} \neq$ $\{\mathrm{id}\}$, then the order of $\phi\left(D_{3}\right)$ is either 1 or 2 , because the group of rotations is the only nontrivial normal subgroup of $D_{3}$; in either case we must have $\phi\left(\rho_{1}\right)=\phi\left(\rho_{2}\right)=\phi\left(\rho_{3}\right)$. On the other hand, if ker $\phi \cap \Lambda \neq\{\mathrm{id}\}$, then because this intersection is invariant under the action of $D_{3}$, it must contain two linearly independent elements $\lambda_{1}, \lambda_{2}$; the group $\Lambda /\left(\lambda_{1} \mathbb{Z} \oplus \lambda_{2} \mathbb{Z}\right)$ is then finite and so is $\phi(\Lambda)$.

Therefore, in order to show that $\phi$ is an isomorphism, it suffices to show that $\left[\eta_{1}\right] \kappa_{f} \neq\left[\eta_{2}\right] \kappa_{f}$ and that $\operatorname{IMG}(f)$ is infinite. The first condition is easily checked by observing that $\mu_{f}\left(\left[\eta_{1}\right]\right)$ and $\mu_{f}\left(\left[\eta_{2}\right]\right)$ are different permutations of $f^{-1}\left(\mathbf{x}_{0}\right)$. The second condition may be seen by restricting our attention to an invariant line such as $\gamma(1)$; on this line $f$ behaves like the single-variable Chebyshev map $s \mapsto s^{2}-2$, and the iterated monodromy group of such a map is known to have elements of infinite order (see [12]).

\section{REFERENCES}

[1] Bartholdi, L., Nekrashevych, V. (2006). Thurston equivalence of topological polynomials. Acta Math. 197(1): 1-51.

[2] Bartolo, E. A., Agustín, J. I. C. (2009). On the topology of hypocycloids. In Mathematical Physics and Field Theory: Julio Abad, in Memoriam. Prensas Universitarias de Zaragoza, 83-98.

[3] Bedford, E., Jonsson, M. (2000). Dynamics of regular polynomial endomorphisms of $\mathbb{C}^{k}$. Amer. J. Math. 122(1): 152-212.

[4] Belk, J., Koch, S. (2010). Iterated Monodromy for a Two-Dimensional Map. In: Bonk, M., Gilman, J., Masur, H., Minsky, Y., Wolf, M., eds. In the Tradition of Ahlfors-Bers V. Contemp. Math. 510: 1-12.

[5] Fornæss, J. E., Sibony, N. (1992). Critically finite rational maps of $\mathbb{P}^{2}$. In: Nagel, A., Stout, E. L., eds. The Madison Symposium on Complex Analysis, Contemp. Math. 137: 245-260.

[6] Godillon, S. (2012). Introduction to Iterated Monodromy Groups. Ann. Fac. Sci. de Toulouse. 21(5): 1069-1118.

[7] Hoffman, M. E., Withers, W. D. (1988). Generalized Chebyshev polynomials associated with affine Weyl groups. Trans. Amer. Math. Soc. 308(1): 91-104.

[8] Hubbard, J. H., Papadopol, P. (1994). Superattractive fixed points in $\mathbb{C}^{n}$. Indiana J. Math. 43(1): $321-365$.

[9] Lopes, A. O. (1990). Dynamics of real polynomials on the plane and triple point phase transition. Math. Comput. Modelling 13(9): 17-32.

[10] Morley, F., Morley, F. V. (2014). Inversive Geometry. Mineola, NY: Dover Publications.

[11] Nakane, S. (2008). External rays for polynomial maps of two variables associated with Chebyshev maps. J. Math. Analysis and Applications 338(1): 552-562. 
[12] Nekrashevych, V. (2011). Iterated monodromy groups. In: Campbell, C., Quick, M., Robertson, E., Roney-Dougal, C., Smith, G., Traustason, G., eds. Groups St Andrews 2009 in Bath, London Math. Soc. Lecture Note Series, vol. 387: 41-93.

[13] Nekrashevych, V. (2012). The Julia set of a post-critically finite endomorphism of $\mathbb{P C}^{2}$. J. Mod. Dyn. 6(3): 327-375.

[14] Ryland, B., Munthe-Kaas, H. (2011). On Multivariate Chebyshev Polynomials and Spectral Approximations on Triangles. In: Hesthaven J., Rønquist E. (eds) Spectral and High Order Methods for Partial Differential Equations. Lecture Notes in Computational Science and Engineering, vol. 76: 19-41.

[15] Uchimura, K. (2001). The set of points with bounded orbits for generalized Chebyshev mappings. Int. J. Bifurcation and Chaos 11(1): 91-107.

[16] Uchimura, K. (2007). Dynamics of symmetric polynomial endomorphisms of $\mathbb{C}^{2}$. Michigan Math. J. $55(3): 483-511$.

[17] Uchimura, K. (2009). Generalized Chebyshev maps of $\mathbb{C}^{2}$ and their perturbations. Osaka J. Math. 46(4): 995-1017.

[18] Van Kampen, E. (1933). On the fundamental group of an algebraic curve Amer. J. Math 55(1): 255-260.

[19] Veselov, A. P. (1986). Integrable polynomial mappings and Lie algebras. In Geometry, Differential Equations and Mechanics (Moscow 1985). Moskov. Gos. Univ. Mekh.-Mat. Fak., 59-63.

[20] Veselov, A. P. (1991). Integrable maps. Russian Math. Surveys 46(5), 1-51.

[21] Withers, W. D. (1988). Folding polynomials and their dynamics. Amer. Math. Monthly 95(5): 399-413.

[22] Zariski, O. (1929). On the problem of existence of algebraic functions of two variables possessing a given branch curve Amer. J. Math 51(2): 305-328. 